\title{
La Biblioteca Central de la Universidad Nacional Autónoma de México a 50 años de su creación: murales vivos, espacios creativos
}

\author{
The central library of the Universidad Nacional Autónoma de Mexico 50 years after its creation
}

\author{
Orlanda Angélica Garrido Yáñes (1), Ramón Trejo González (2) \\ y Gloria Adriana Hernández Sánchez (3)
}

(1) Dirección General de Bibliotecas, Edificio de Biblioteca Central, Circuito Interior s/n México D. F., Coyoacán C.P. 04510 orlanda@dgb.unam.mx (1) ramtrejo@dgb.unam.mx (2) ahdz@dgb.unam.mx (3)

\begin{abstract}
Resumen
A los 51 años de haber abierto sus puertas, la Biblioteca Central se define como sede del Sistema Bibliotecario de la Universidad Nacional Autónoma de México (UNAM) y como biblioteca central pública en la denominada Ciudad Universitaria. Se encuentra ubicada estratégicamente en un lugar accesible para la comunidad universitaria. El espacio comprende una superficie construida de $10,000 \mathrm{~m} 2$ en un edificio de 16 Pisos. El propósito de esta comunicación es mostrar de manera sucinta las variaciones y transformaciones del edificio de Biblioteca Central durante 50 años en cuanto a su distribución interna, para concluir con las últimas adecuaciones a fin de obtener mayor funcionalidad del edificio para los acervos y áreas, espacios para el personal y mayor capacidad de movilidad para los usuarios que día con día alcanzan en promedio las 8,000 asistencias.
\end{abstract}

Palabras clave: Bibliotecas Universitarias. Espacios para bibliotecas universitarias. Edificios para bibliotecas. Accesibilidad.

\section{Introducción}

Desde el 5 de abril de 1956, la Biblioteca Central abrió sus puertas y espacios a la comunidad de la Universidad Nacional Autónoma de México (UNAM).

El edificio que alberga la Biblioteca Central tuvo como propósito original servir de sede a la $\mathrm{Bi}$ blioteca y Hemeroteca Nacionales. Esta idea fue rechazada por miembros de la Cámara de Senadores e intelectuales del país que finalmente decidieron crear la Biblioteca Central de Ciudad Universitaria.

Exteriormente, en una superficie de cuatro mil metros cuadrados. Juan O'Gorman, pintor y arquitecto, autor del diseño y los motivos del mural y quien participó en la edificación del inmueble, decidió utilizar mosaicos de piedras de colores para hacer ornamentalmente

\begin{abstract}
51 years after having opened its doors, the Central Library is the seat of the Library System of the UNAM and a central public library denominated University City Library. It is located strategically in an accessible place for the university community. The space includes a constructed surface of $10.000 \mathrm{~m} 2$ in a building of 16 Floors. The intention of this communication is summarize the variations and transformations of the building of Central Library during 50 years as far as its internal distribution, to conclude with the last adjustments, designed in order to obtain greater functionality of the building for the holdings and services, spaces for the personnel and allow for greater user mobility, who reach in average the 8.000 daily attendances.
\end{abstract}

Keywords: University libraries. Spaces for university libraries. Buildings for libraries. Accessibility.

colores para hacer ornamentalmente atractiva la arquitectura del edificio y plasmó en sus fachadas la cultura mexicana representada por importantes épocas; período prehispánico, la conquista, el virreinato, la independencia, la revolución y la época contemporánea, considerada esta última hasta el momento en que se realizó la obra. A decir de O'Gorman, fue el primer gran edificio mexicano construido ex profeso para albergar en sus dieciséis niveles el saber nacional y universal.

\section{Desarrollo histórico}

Al interior, en un periódico de circulación nacional en el año de 1952, cuatro años antes de abrir, se detallaba que la Biblioteca Central tendría una planta baja, un vestíbulo con espacio para exposiciones, sala de lectura general co- 
nectada directamente con un jardín, un salón para catalogar los libros, una oficina de control y préstamo y hacia el lado norte un pequeño salón de conferencias y una pequeña escuela de bibliotecarios. La amplitud del edificio permitiría tener un restaurante, una sala de exposiciones permanentes y tres salones para seminarios. En la planta alta se localizará la colección de mapas y la discoteca (con servicio de grabaciones de todas las conferencias de interés general que se efectúen en Ciudad Universitaria). Finalmente, con un costo aproximado equivalente a cuatrocientos mil euros se realizó la construcción para albergar 1.200.000 libros, es decir, ciento veinte mil por piso. No obstante, inició con un fondo bibliográfico de veinte mil títulos únicamente y con servicio de estantería cerrada.

Se iniciaron las actividades con una plantilla de 56 empleados en un horario de doce horas, es decir, de 8:00 a 20:00 hrs.

En sus inicios estuvo del Departamento Técnico, la Coordinación de Bibliotecas de escuelas y facultades y los servicios al público de la colección inicial. Diez años más tarde se crearía la Dirección General de Bibliotecas que hasta la fecha se mantiene en el edificio. La Biblioteca Central también fue sede por 24 años del Colegio de Bibliotecología de la Facultad de Filosofía y Letras de la UNAM.

En un principio, su estructura albergó tres departamentos: procesos técnicos, servicios al público y coordinación de bibliotecas. Cada departamento a su vez tuvo varias secciones. Procesos técnicos estaba dividido en catalogación y distribución, adquisiciones y clasificación; el departamento de servicios al público en consulta, publicaciones periódicas, préstamos, libros en reserva y material audiovisual.

La sala de lectura se ubicó en el piso principal del edificio con una superficie de $1800 \mathrm{~m} 2$, incluida una pequeña parte para el elevador. En los pisos superiores denominados entrepiso y planta alta, se destinaron cubículos para investigadores, sala para conferencias, mapoteca, discoteca, linguafón y dirección. Los pisos superiores sirvieron para almacenar libros. Sin embargo, es en 1983 cuando se abre el acervo al público. Para 1960 la Biblioteca Central contaba con 40,000 volúmenes.

Diecisiete años después, algunos pisos del edificio de la Biblioteca Central tuvieron otros usos: institutos y facultades de la UNAM guardaban equipo y mobiliario obsoleto; la Biblioteca Nacional disponía de un piso completo para bodega; por otra parte se albergó el Colegio de Bibliotecología de la Facultad de Filosofía y Letras y el Centro de Estudios Literarios que actualmente es el Instituto de Investigaciones Filológicas y mantiene a la fecha una cuarta parte del ala oriente de la planta alta dividida en cuatro cubículos, ocupados por un investigador emérito.

Para 1961 se adquirieron 15 mil 18 volúmenes y se atendieron 257 mil 352 lectores. Para 1962 se alcanza la cifra de 60 mil volúmenes y se monta la $1^{\mathrm{a}}$ exposición permanente "La universidad de ayer y hoy".

En 1963 la Biblioteca Central dispone de 70 mil volúmenes y agrega una sala denominada de "Joyas Bibliográficas" para los investigadores. Se considera un laboratorio de desinsectización y de reparación de libros y documentos y un amplio local adaptado para exposiciones de libros y otros elementos gráficos relacionados con el libro.

Hacia el año de 1964 se definen las áreas de Biblioteca Central, Joyas Bibliográficas, Exposiciones, Archivos, Historia de la Universidad de México, Organización Biblioteconómica, así como las secciones de tesis graduados, Canje y Donaciones, Fotocopia y Microfilm así como Exposiciones e Información a visitantes. En el $8^{\circ}$ piso se reinstaló el Archivo Histórico.

Al año siguiente se puede disponer del préstamo a domicilio e interbibliotecario así como del servicio de fotocopiado y de un aparato para micropelículas.

En el año de 1966 se inicia el cambio de estantería de madera por metálica, en el primer piso.

Desde 1967 se distribuyen las áreas en los 16 niveles: en los sótanos uno y dos la Distribuidora de Libros Universitarios, que los utilizó como bodega de sus librerías, una de ellas en funcionamiento en el propio edificio en ese tiempo; en el basamento, Adquisiciones, el departamento técnico, el laboratorio de restauración, la imprenta, el laboratorio de fotografía y el comedor para el personal; en el primer piso el acervo general; segundo piso la librería universitaria; tercer piso, publicaciones periódicas; cuarto piso, tesis; quinto piso, canje y donación; sexto y séptimo pisos, acervos embodegados de la Biblioteca Nacional; octavo piso, archivo histórico; noveno piso, colección de libros raros y valiosos; planta alta el colegio de bibliotecología y archivología y el centro de estudios literarios con su biblioteca "Julio Jiménez Rueda"; y en el entrepiso la dirección general, planeación, diversas oficinas administrativas y el centro de documentación que funcionó de 1969 a 1978.

A la par de las modificaciones estructurales, en 1975 la Biblioteca Central se constituye en el Departamento de Servicios al Público; en 1976 ingresa la primer plantilla de personal académi- 
co y en ese mismo año el $8^{\circ}$ piso es desocupado debido a que el Archivo Histórico se integra al Centro de Estudios sobre la Universidad.

\begin{tabular}{|c|c|c|}
\hline \multirow{2}{*}{ NIVEL } & \multicolumn{2}{|c|}{ RESUMEN } \\
\hline & $\mathrm{BC}$ & DGB \\
\hline $10^{\circ}$ & 359 & 359 \\
\hline $9^{\circ}$ & & 718 \\
\hline $8^{\circ}$ & 718 & \\
\hline $7^{\circ}$ & 718 & \\
\hline $6^{\circ}$ & 718 & \\
\hline $5^{\circ}$ & 718 & \\
\hline $4^{\circ}$ & 718 & \\
\hline $3^{e r}$ & 718 & \\
\hline $2^{\circ}$ & 718 & \\
\hline $1 \mathrm{er}$ & 718 & \\
\hline P. ALTA & & 1093 \\
\hline P. MEZANINE & 400 & 1200 \\
\hline P. PRINCIPAL & 1985 & \\
\hline P. BASAMENTO & 385 & 1600 \\
\hline SOTANONO 1 & 100 & 265 \\
\hline SOTANOO 2 & 365 & \\
\hline TOTAL & 9338 & 5235 \\
\hline$\%$ & $64.08 \%$ & $35.92 \%$ \\
\hline
\end{tabular}

Figura 1. Edficio de la Biblioteca Central: $\mathrm{m}^{2}$ por niveles)

\begin{tabular}{cc}
\hline Piso & Personal \\
\hline $10^{\circ}$ & 5 \\
\hline 9 & 20 \\
\hline 8 & 12 \\
\hline 7 & \\
\hline 6 & 12 \\
\hline 5 & 12 \\
\hline 4 & 12 \\
\hline 3 & 12 \\
\hline 2 & 12 \\
\hline 1 & 12 \\
\hline PA & 20 \\
\hline E & 48 \\
\hline PP & 30 \\
\hline Basamento & 173 \\
\hline Sótano 1 & 3 \\
\hline Sótano 2 & 1 \\
\hline
\end{tabular}

Figura 2. Distribución del personal por pisos

Para 1977 se adquirieron 6,858 volúmenes. Para 1978 hay un promedio de 1690 lectores por día y el préstamo de libros alcanza la cifra mensual de 41,733. Se renueva la estantería de la sala de consulta general.

Por otra parte, para 1979 el promedio de lectores por día es de 1709 .

Durante 1980 se busca solucionar el acondicionamiento de las oficinas de la Dirección General
Bibliotecas en el edificio: áreas técnicas, académicas y administrativas. Se incorporan escaleras y elevadores para introducir a los usuarios a las áreas de acervo en los pisos superiores.

En 1981 la administración en turno considera importante revisar los hábitos y concepción necesaria para modificarse. Presentan las Bases para la Racionalidad del Sistema Bibliotecario Universitario y enfrenta de lleno la remodelación de la Biblioteca Central. En diciembre de 1981 se autorizan las tareas de remodelación del inmueble y salen las oficinas del edificio, los servicios se suspenden y mediante la negociación con los trabajadores se reacomoda casi un millón de libros y más de 8 mil estantes. El proyecto se denomina "Nueva biblioteca central" y pugna por la estantería abierta en colección general y consulta y por la mixta en la colección de tesis.

Así, se replanteó el sentido de la Biblioteca Central, dada la problemática y extensión del Sistema Bibliotecario de la UNAM.

En abril de 1983 reabre sus puertas con una nueva forma de organización, como prestadora de servicios; en ese tiempo se realiza la microfilmación de 12 mil tesis.

Para 1984 se cuenta con 6 departamentos: préstamo de libros, consulta de tesis, publicaciones periódicas, selección y adquisición bibliográfica, organización y métodos y un centro de documentación para el posgrado.

Con la automatización de servicios en 1986 se instala un aparato detector de cintas magnéticas para evitar el robo de libros. Se atienden 27 mil usuarios en este periodo.

En 1987 se rescatan del sótano del edificio 11229 volúmenes y 5000 fascículos.

De 1984 a 1988 se ingresan 51 mil volúmenes y se atiende a 2.5 millones de usuarios.

A partir de 1989 la UNAM emprende un Programa de Modernización de Bibliotecas que incluye incremento de acervos, adecuación de espacios, estanterías abiertas, ampliación de horarios y mayor automatización en los servicios.

A fines de 1999 y principios de 2000, tras casi un año de paro estudiantil y después del compromiso de contribuir a la normalización de la vida universitaria, la administración en turno tiene como objetivo devolver a la Biblioteca Central su carácter de biblioteca modelo rescatando el uso de espacios, ampliando su horario de trabajo, crear nuevos espacios y modernizar los existentes. 
Entre 2000 y 2001 la administración en turno recurre al Plan Maestro del Patrimonio Inmobiliario a cargo de la Dirección General de Obras y Conservación de la UNAM, elemento que permite regular y normar de manera integral y ordenada el crecimiento, adecuación y optimización de la planta física de la universidad. En consecuencia, el arquitecto Luis Fernando Solís, es el encargado del Plan Maestro Inmobiliario de la Dirección General de Bibliotecas y Biblioteca Central y su objetivo principal se centró en la optimización de los recursos y el logro de un adecuado funcionamiento y aprovechamiento de las instalaciones físicas.

Las expectativas para la Biblioteca Central tuvieron como propósito reordenar las áreas ocupadas anárquicamente, reagrupar espacios según las funciones y lograr que el crecimiento a futuro fuera ordenado; en tanto, para la Dirección General de Bibliotecas la reubicación de las áreas —dado el desarrollo constante-, preveía una posible construcción de un nuevo edificio para permitir su progreso y crecimiento.

La metodología del Plan Maestro incluyó entre sus objetivos el detectar necesidades generadas tanto por la demanda de usuarios como su futuro crecimiento para lograr en un mínimo período, el óptimo aprovechamiento del espacio con base en una adecuada administración de su plantilla física; realizar un análisis de la ocupación para ordenarla de modo que se conservara su destino original; fijar los límites de crecimiento para el buen funcionamiento de la planta física e implantar la instalación de circulaciones, estacionamientos y sanitarios apropiados para los usuarios con discapacidad.

A través de los años, con el crecimiento de la población estudiantil en la universidad, el edificio de Biblioteca Central perdió su finalidad primordial. La convivencia de dos organismos en un mismo edificio Biblioteca Central y Dirección General de Bibliotecas, ocasionó problemas serios en la utilización de espacios. En su concepción, el acervo quedó protegido de la luz solar y el polvo en los pisos superiores pero con el tiempo se pasó a la planta baja y actualmente no se tiene la opción de regresar la pesada carga debido a las condiciones estructurales del edificio y las normas del reglamento después del sismo ocurrido en la Ciudad de México en 1985.

Asimismo, en el diagnóstico inicial del Plan Maestro se detectaron varias situaciones en el interior:

- en un alto porcentaje en las áreas se carece de iluminación y ventilación natural y es nece- sario reubicarlas pues son espacios de oficina y enseñanza;

- dada la antigüedad del edificio, gran parte de la herrería presenta oxidación;

- los acabados del piso en general están en muy mal estado, originalmente fueron de loseta de asfalto que acabó por romperse y desprenderse; algunas áreas fueron cubiertas con alfombra;

- en lo tocante a las instalaciones eléctricas, se observan fallas debido a la longevidad del equipo;

- en lo correspondiente a las instalaciones hidrosanitarias, se observaron graves desperfectos en la red hidráulica y sanitarios debido al envejecimiento normal de las piezas;

- los equipos de aire acondicionado producen ruido ya que no fueron renovados y no fueron ocultados en la azotea del edificio dando un mal aspecto;

- el deterioro del mobiliario y falta de confort;

- pese al tiempo, al desgaste natural del viento, la lluvia, el sol y los cambios de temperatura, los murales se han conservado, aunque requieren de mantenimiento, pues se han desprendido piedras que pueden ser insustituibles;

- en la parte inferior de las fachadas, el edificio se ha visto atacado con grafiti y pintas, sobre todo en la cantera ya que absorbe la pintura;

- asimismo, el ónix del área de lectura de la planta principal requiere restauración mayor por el agrietamiento que presenta.

Entre julio y agosto de 2000 , se remodela la planta principal que recupera su luminosidad, se rehabilita la fuente externa y se abre el acceso norte de la biblioteca. El edificio recupera gran parte de su diseño original, se renueva el mobiliario hasta donde los recursos lo permiten. El pormedio diario de asistencia es de 7 mil usuarios. En la planta principal se rehace el mostrador y las oficinas de Biblioteca Central.

En 2001 continúan las remodelaciones del edificio, las áreas administrativas ubicadas en el basamento de la torre. Se remoza el núcleo de sanitarios y se da mantenimiento a las escaleras de cantera en la entrada norte. En este mismo año se presenta una exposición y se recupera la tradición de concebir la Biblioteca Central como un recinto para fomentar y difundir la cultura.

En el rescate del Fondo Antiguo se divide el acervo en: Tesoros Bibliográficos (1500-1799) y Moderna (1800-1950). En este mismo año se 
realiza un inventario del acervo. La Biblioteca Central atiende a 2.394.336 usuarios.

Se reordena el ala oriente de la planta principal y se reduce el mostrador de atención gracias a la incorporación de computadoras y lectoras ópticas. En la sala de lectura había una saturación de estantería que impedía a los usuarios el aprovechamiento de la luz natural de los tragaluces y vitrales en las ventanas.

En 2002 se amplía el horario de servicios: todos los días incluyendo días festivos de 8:30 a 21:30 hrs. Asimismo, presenta una nueva imagen exterior el edificio, pues iluminan las cuatro caras del mural realizado por O'Gorman. Se atiende a 2,488 054 usuarios. En este tiempo se remodela el departamento de Consulta y el área de fotocopiado en la planta principal; en el ala oriente de basamento se remozan los departamentos de Procesos Técnicos, Adquisiciones y Restauración; del área poniente el departamento de Selección de Adquisiciones Bibliográficas, la Unidad Administrativa, la imprenta y se rehabilita una fuente interna. En el entrepiso se reacondiciona el núcleo de sanitarios.

En 2003 se atiende a 2.962 .042 usuarios. Las remodelaciones arquitectónicas prosiguen tanto en áreas administrativas, Procesos Técnicos y Adquisiciones Bibliográficas, como en áreas abiertas a los usuarios y la renovación de la sala de consulta electrónica, equipada con $50 \mathrm{com}$ putadoras. En este mismo año se inicia la Campaña Financiera Pro-Remodelación y Equipamiento de la Biblioteca Central, con el respaldo de la Fundación UNAM y se monta en el jardín de la Biblioteca Central, por primera vez en su historia, la feria del libro de Biblioteca Central. Se rehabilita la escalera de acceso a la sala de consulta electrónica y en la planta principal el elevador interno para empleados. En la parte del basamento se remoza el núcleo de sanitarios y se inicia el acondicionamiento del Centro Estructurado de Voz y Datos.

En 2004 se proyectan las etapas de remodelación del inmueble, iniciándose los trabajos para una sala de lectura modelo la Biblioteca Central, el diseño de 1 sala de educación a distancia y 2 salas de videoconferencias con capacidad para 20 personas, equipadas con la más avanzada tecnología audiovisual y de telecomunicaciones. Se habilitan las aulas de usos múltiples y cómputo en el décimo piso y en el noveno unas oficinas administrativas.

Para el año 2005 se reubica el cubículo sindical en el sótano uno y se continua con la confección de los cuartos de voz y datos para catorce pisos.
En el 2006, en el primer piso se acondiciona la sala modelo de acervos y videoteca; en el basamento se concluye la sala de videoconferencias y de educación a distancia; en el noveno piso se instala aire acondicionado; para el octavo piso se instalan contactos y cableado de corriente regulada así como aire acondicionado. Por la parte exterior del edificio, en la rampa exterior se instala una planta de luz de emergencia y su ventilador; en la planta principal se inicia la impermeabilización de cantera en la entrada sur, se acondiciona el control pacífico de aves y se instala una película protectora de blindaje de cristales.

Para el año 2007, en la planta alta lado oriente se remodelan las oficinas de la Subdirección de Planeación que se integran al área que ocupa el departamento de Planeación y se genera un nuevo servicio en el jardín interior de la biblioteca denominado jardín cibernético donde se tuvieron que instalar cinco mesas con conexiones eléctricas y red inalámbrica con el propósito de brindar a la comunidad un espacio mayor de navegación.

Pese a la escasa información que relate los cambios internos y externos del edificio, es patente a través del tiempo que se puede contemplar la compleja coexistencia de Biblioteca Central primero con otras instancias universitarias y posteriormente con la Dirección General de Bibliotecas en un espacio que de origen pertenece a Biblioteca Central. La competición por los espacios y la privación de los mismos día a día se ha incrementado y la Biblioteca Central ha cedido más áreas ante la incesante expansión de la Dirección General de Bibliotecas; con ello ha visto mermado su lugar, lo que dificulta la consecución óptima de los proyectos que como biblioteca universitaria tiene trazados.

No obstante lo anterior, se ha avanzado poco a poco y a través de pequeñas acciones en la reutilización de espacios desaprovechados y más con intuición que con recursos económicos, pues se precisa solucionar infinidad de aspectos de urgente atención hasta detalles de funcionamiento y comodidad.

Estas mejoras en el edificio garantizan conservar a la Biblioteca Central a la vanguardia de los servicios bibliotecarios en México, permitiéndole contribuir con una entidad viva, dinámica y actual. Las remodelaciones han dignificado de fondo este edificio cincuentenario. Las tareas estratégicas son incesantes. 


\section{Conclusiones}

- Si bien exteriormente el edificio no ha sufrido modificaciones significativas ni expansiones debido a su estructura que lo hace único, interiormente se ha remodelado al paso de los años, adecuando los espacios a las crecientes necesidades y servicios que demanda la comunidad universitaria.

- No obstante, se debe contemplar que para la satisfacción de nuevas necesidades de un inmueble de esta naturaleza, se requiere mantener un total respeto ante la integridad del edificio como obra artística y arquitectónica.

- De acuerdo al Plan Maestro es preciso aplicar criterios de re-arquitectura, lo que significa no solo restaurar o remodelar, sino respetar el proyecto inicial y el elemento tiempo que modifica el uso, el destino y las funciones, lo que permitirá reutilizar un edificio sin forzar su concepto original.

- Se contempla la posibilidad de reubicar las áreas académicas y administrativas de la Dirección General de Bibliotecas en un nuevo edificio ya que actualmente ocupan el $36 \%$ de espacios que la Biblioteca Central requiere para el albergar el acervo y proporcionar diversos servicios. Con esta opción se aumentará la capacidad de espacio y su óptima distribución.

- El mantenimiento de un inmueble de esta naturaleza es costoso y requiere de una planeación estratégica y aplicación de normas para acondicionarlo de acuerdo a la exigencia de los servicios en un tiempo breve, con mínimas incomodidades e interrupciones tanto para el personal como para los usuarios.

\section{Referencias}

Biblioteca Central: libros muros y murales: $50^{\circ}$ aniversario (2006). Celia Martín Marín, [et al], México: UNAM, Dirección General de Bibliotecas, 2006, 256 p.

Solís Ávila, Fernando (2001). Plan maestro inmobiliario [Documento de trabajo]. México: Dirección General de Obras UNAM, 2001. 108 p. 